\title{
Modeling and Simulation of the Autocatalytic Kinetics of Haemoglobin SS Polymerization: Onset of Polymerization
}

\author{
Edith Egbimhanhu Alagbe1, Alfred Akpoveta Susu2 ${ }^{2}$ Adedoyin Owolabi Dosunmu ${ }^{3}$ \\ ${ }^{1}$ Department of Chemical and Polymer Engineering, Lagos State University, Lagos, Nigeria \\ ${ }^{2}$ Department of Chemical Engineering, University of Lagos, Lagos, Nigeria \\ ${ }^{3}$ Department of Hematology, Lagos State University Teaching Hospital, Ikeja, Lagos, Nigeria \\ Email: alfredasusu222@hotmail.com
}

Received 5 November 2015; accepted 10 March 2016; published 17 March 2016

\begin{abstract}
We report a fresh and simpler approach to the modelling of the kinetics of the polymerization of Hb SS in sickle cell patients that couples the kinetics and the hydrodynamics of blood flow in mechanistic understanding of the process. The well-known two-step autocatalytic reaction scheme was used for the polymerization reaction with the assumption of simpler first-order reaction scheme for each stage. In addition, the forces acting on a particle in motion were also introduced to account for compelling settling of the red cells that lead to vessel occlusion (vaso-occlusion). A first attempt on the prediction of vessel blockage was made using this novel model. The time for the onset of the polymerization reaction was derived from hydrodynamic considerations and kinetics while the kinetic rate constants were obtained from the autocatalytic nature of the reaction. Experimental data for model validation were obtained from recruited SS patients and in vitro data of Hofrichter. Over 100 volunteers were recruited for participation in this work but less than $\mathbf{4 0 \%}$ met the inclusion criteria. Participants were of age range 13 - 43 (with a mean of $26 \pm 8$ years) for SCD patients and 18 - 43 (with a mean of $28 \pm 7$ years) for control participants. Blood indices and Transcranial Doppler (TCD) test parameters of all participants were the principal parameters used for model validation. Constant $k_{2} / k_{1}$ ratios was obtained for individual in vivo/in vitro system. This ratio is unique for any individual, independent on protein sequence and also suggests the degree of expression of the symptoms of Sickle Cell Disease (SCD) with higher values reflecting greater propensity to pain crisis. Delay time, $t_{D}$, was found to have an inverse relationship with the kinetic constant for the residual reaction, $k_{1}$. Therefore, long delay times calculated, offer insight on why SCD patients are not in perpetual crises because enough time is provided the cells to escape microcirculation while keeping the residual reaction at the minimum. Sensitivity analysis was carried out to obviate the limitations encountered in the course of the work. Results showed the onset of occlusion to be most sensitive to the diameter of the blood vessel.
\end{abstract}

\section{Keywords}

Vaso-Occlusion, Hb SS Polymerization Kinetics, Residual and Secondary Reactions, Hydrodynamics of Blood Flow, Onset of Hb SS Polymerization 


\section{Introduction}

The sickle haemoglobin was identified as the first protein to cause disease (Pauling L, Itano HA, Singer SJ, Wells IC, 1949) [1]. The reason for this defect is the replacement of a negative glutamic acid (Glu) molecule with a hydrophobic valine (Val) molecule on the two beta subunit of the haemoglobin. While the former is able to make hydrogen bond and dominate the interactions in which they participate, the latter avoids contact with water (in the blood, in this case) and pack against each other (Petsko GA, Ringe D, 2004) [2].

Sickle cell disease (also known as Sickle Cell Anemia) is characterized by a molecular hemoglobin defect which causes the polymerization of deoxygenated hemoglobins and results in reduced erythrocyte flexibility, deformation and numerous rheological effects. Sickle cell anemia produces an abnormal type of hemoglobin called hemoglobin S (Hb S), which has less oxygen-carrying capacity. SCD is a blood disorder characterized by abnormal hemoglobin in the red blood cell. The red blood cell, RBC, has a diameter of about $7 \mu \mathrm{m}$ and due to their flexibility are able to pass through capillaries of $\leq 3 \mu \mathrm{m}$ diameter. In the case of SCD, the red blood cells have lowered deformability due to repeated sickling (polymerization) and unsickling (melting) processes and eventually become irreversibly sickled and have to be removed from circulation. This happens frequently and accounts for the anemia characteristic of the disease. Occlusion of vessels is a common phenomenon as these sickled cells co-operate to form aggregates that later block the vessels.

Sickle haemoglobin polymerization appears to be precise with respect to location and directionality of growth. Usually, thermal fluctuations provide the energy required for molecules of sickle hemoglobin that are competent to form long fibers, to assemble into small aggregates. This leads to the spontaneous formation of transient species of various sizes. At some critical size (called the nucleus), each additional molecule lowers the energy of the aggregate. The formation of nuclei is thus characterized by molecular fluctuations, and the time to form a nucleus is inherently stochastic.

All of the kinetic findings can be explained by a novel nucleation mechanism which postulates that the first polymer in a given solution volume forms via a simple homogeneous nucleation mechanism. This polymer grows by the addition of monomers to the end. The lateral surface of the growing oligomers may also serve as a template for the nucleation of new polymers (Heterogeneous nucleation).

Therapeutic options available from kinetic studies and currently in use in some cases, are: In particular, the discovery of the enormous concentration dependence of the rate of polymerization suggested decreasing the intracellular haemoglobin concentration as a new approach to the treatment of sickle cell disease; Decreasing the intracellular haemoglobin concentration (by crowding) with non-polymerizing agents like fetal haemoglobin $\mathrm{Hb}$ F or normal Hb AA (as currently used in Stroke Prevention Trial in Sickle Cell Anemia, STOP). A decrease in the intracellular haemoglobin concentration of only $10 \%-15 \%$ has been predicted to have some therapeutic effect as evidenced in blood exchange therapies; Increasing the oxygen affinity so that the concentration of molecules capable of polymerization will be grossly decreased; Drugs that will prevent cellular dehydration.

\section{Methodology}

A sample size of 30 was obtained using the formula (FAO, 1990; Magnanni, Robert, 1997; UNICEF, 1995): [3]-[5]

$$
n=\frac{t^{2} p(1-p)}{m^{2}}
$$

where:

$n=$ required sample size.

$t=$ confidence level at 95\% (Standard value of 1.96).

$p=$ Estimated prevalence of variable under investigation (SCD) in the project area.

$m=$ Margin of error at $5 \%$ (standard value of 0.05 ).

\subsection{Study Area and Population}

Lagos metropolis was used as study area for this project. It was a cross sectional observational study. The study population was sickle cell patients within the age of 13 years and 45 years and Controls with hemoglobin AA and AS (age and sex matched) attending the paediatric and adult hematology clinics of the Lagos State Univer- 
sity Teaching Hospital. Written consent were obtained from patients and their parents (for ages that are less than 18 years) after due explanation.

\subsection{Research Procedures}

Participants were invited to the clinic for recording of history, physical examination, laboratory and TCD tests. Thereafter, study questionnaires were filled and signed by all participants. The exclusion criteria was used to further filter out eligible participants for the Research study. Inclusion criteria were that $\mathrm{Hb}$ SS patients in the study must be in steady state, that is, patients that are not in any form of crisis. The controls were obviously normal $\mathrm{Hb} \mathrm{AA}$ and $\mathrm{Hb}$ AS individuals with age and sex matching those of the sickle cell patients while Exclusion criteria were patients and controls with any comorbid chronic infection and obvious vascular abnormality.

Blood indices and Transcranial Doppler on the Anterior Carotid Artery, ACA; Internal Carotid Artery, ICA and Middle Cerebral Artery, MCA were carried out in all 21 SCD and 10 Control participants.

\section{Model Development}

\subsection{Autocatalysis of the Hb SS Polymerization}

If $A=$ hemoglobin, $\mathrm{Hb} ; B=$ oxygen and $C=\mathrm{Hb}$. $\mathrm{O}_{2}$ polymer, we present the autoctatlytic scheme of (Boudart M., 1968) [6] and (Susu AA, 1997; Susu AA, Kunugi T, 1980) [7] [8] to obtain:

$$
\begin{aligned}
& A+B \rightarrow C \\
& A+B \stackrel{C}{\longrightarrow} C
\end{aligned}
$$

In terms of reaction rate, the equations are represented as:

$$
\begin{aligned}
& r_{1}=-\frac{\mathrm{d} C_{A}}{\mathrm{~d} t}=k_{1} C_{A} \\
& r_{2}=-\frac{\mathrm{d} C_{A}}{\mathrm{~d} t}=k_{2} C_{A} C_{C}
\end{aligned}
$$

The overall reaction rate, $r_{0}$ becomes:

$$
r_{0}=r_{1}+r_{2}=r_{0}=k_{1} C_{A}+k_{2} C_{A} C_{C}
$$

In terms of fraction converted, Equation (3.5) becomes,

$$
r_{0}=-\frac{\mathrm{d} C_{A 0}(1-f)}{\mathrm{d} t}=k_{1} C_{A 0}(1-f)+k_{2} C_{A 0}(1-f) C_{A 0} f
$$

Rearranging and setting $k=k_{2} C_{A 0}$ and $\rho=k_{1} / k$ gives:

$$
\frac{\mathrm{d} f}{\mathrm{~d} t}=k(f+\rho)(1-\mathrm{f})
$$

Setting the second derivative of Equation (3.8) to zero gives the point of inflection for the function and the maximum rate occurs at $f=1 / 2$

Also, integrating Equation (3.8) with $f=0$ at $t=0$, we have;

$$
f=\rho \frac{\operatorname{eexp}[(1+\rho) k t]-1}{1+\rho \exp [(1+\rho) k t]}
$$

Taking $\ln$ of both sides and precipitating out $t_{1 / 2}$ at the point of maximum rate, where $f=0.5$, Equation (3.9) becomes:

$$
t_{1 / 2}=\frac{1}{(1+\rho) k} \ln \left(\frac{1+2 \rho}{\rho}\right)
$$

From Equation (3.10), the rate constants for the reaction can be obtained. 


\subsection{Model for Settling Velocity of the RBC}

The total amount of force exerted on a particle can be summarised as:

Force due to Acceleration, $F_{a}=$ Gravity Force, $g$-Buoyancy Force, $F_{b}$-Drag Force,

$$
F_{d}
$$

Therefore, from Equation (3.11), for a particle to settle (attain its terminal velocity), $F_{b}$ must be equal to $F_{d}$. So, for many spheres, it is represented as:

$$
F_{b}=g V_{p}\left(\rho_{p}-\rho\right)(1-\phi)=F_{d}=\phi S \frac{1}{2} \rho u_{s}^{2}
$$

where $\rho_{P}=$ density of particle $=$ MCHC value from clinical analysis, $\rho=$ density of fluid (serum), $g=$ gravitational constant, $V_{p}=$ volume of particle = Actual red cell volume, $\phi=\frac{N V_{p}}{V}=$ hct, $S=$ drag co-efficient, $u_{S}=$ settling velocity. Settling velocity from Equation (3.12) is:

$$
u_{S}=\sqrt{\frac{2\left(\rho_{P}-\rho\right)(1-\phi) g v_{P}}{S \phi \rho}}
$$

\subsection{Prediction of Time of Vaso-Occlusion in the Vessel}

For a fluid flowing through a pipe, the Poiseuille equation (Charm SE, Kurland GS, 1974) [9] is used to describe the plasma velocity, $u_{P}$, such that:

$$
\frac{\mathrm{d} u_{P}}{\mathrm{~d} t}=\frac{\pi}{8}\left(\frac{R^{4}}{\mu_{0}}\right)\left(\frac{P_{1}-P_{2}}{L}\right)
$$

Separating variables here and integrating between limits yields:

$$
\int_{u_{\mathrm{s}}}^{u_{p}} \mathrm{~d} u_{p}=\frac{\pi R^{4} \Delta P}{8 \mu_{0} L} \int_{0}^{t_{1} / 2} \mathrm{~d} t
$$

Therefore,

$$
t_{1 / 2}=\frac{8 \mu_{0} L u_{s}}{\pi R^{4} \Delta P}\left(u_{p}-u_{s}\right)
$$

The time for the onset of pain threshold in sickle cell patients is given by Equation (3.15).

\section{Results and Discussion}

\subsection{In Vitro Results}

For the in vivo data, with the assumption that onset of critical occlusion time corresponds to the time of maximum reaction, the $k$ values (and corresponding slopes) at the points of inflection and occlusion time were computed for all arteries under investigation from Equations. (3.13), (3.44) and (3.15) using MatLab programming. The results obtained are shown in Table 1.

For all data presented here, $t_{1 / 2}=$ time to reach $50 \%$ conversion, min; $S=$ slope at the point of inflection (that is, at $50 \%$ conversion) $=$ Number of nuclei formed per time per volume, $\min ^{-1} \cdot \mathrm{L}^{3} ; t_{D}=$ delay time, $\min ; K=$ $k_{2} C_{\mathrm{A} 0}, \mathrm{gL}^{-1} \cdot \mathrm{min}^{-1}, k_{1}=$ rate constant for the residual (homogeneous) reaction, $\min ^{-1}, k_{2}=$ rate constant for the heterogeneous polymerisation, $\min ^{-1}$.

For in vitro data, relevant data like the values of slope, time to reach $50 \%$ conversion and delay times were extracted from the curves of previous works of (Hofrichter J, Ross PD, Eaton WA, 1974) [10] from calometric and optical birefringence measurements of the time course of sickle cell polymerization. Values of the slope and time to reach 50\% conversion were inserted into Equations. (3.13) and (3.15). MATLAB programming was used to obtain the values of $k_{1}$ and $k_{2}$ at different temperatures. The results obtained are presented in Table 2 and Table 3. 
Table 1. Comparing $k$ values in both sides of the skull (subscript 1 and $2=$ Residual and secondary rate constants respectively).

\begin{tabular}{|c|c|c|c|c|c|c|c|c|c|c|c|c|}
\hline & LEFT & & & & & & RIGHT & & & & & \\
\hline & ACA & & ICA & & MCA & & ACA & & ICA & & MCA & \\
\hline CODE & $k_{1}$ & $k_{2}$ & $k_{1}$ & $k_{2}$ & $k_{1}$ & $k_{2}$ & $k_{1}$ & $k_{2}$ & $k_{1}$ & $k_{2}$ & $k_{1}$ & $k_{2}$ \\
\hline 101 & 14.392 & 39.539 & 279.901 & 768.959 & 22.219 & 61.042 & 35.981 & 98.848 & 279.901 & 768.959 & 44.438 & 112.083 \\
\hline 102 & 25.052 & 68.262 & 365.358 & 995.527 & 34.809 & 94.848 & 75.155 & 204.781 & 292.314 & 796.495 & 13.924 & 37.939 \\
\hline 103 & 23.889 & 65.991 & 371.711 & 1026.83 & 19.67 & 54.336 & 95.555 & 263.963 & 371.711 & 1026.83 & 17.703 & 48.903 \\
\hline 104 & 43.615 & 118.519 & 282.702 & 768.213 & 161.594 & 439.113 & 87.23 & 237.038 & 339.34 & 922.119 & 32.32 & 87.826 \\
\hline 105 & 36.463 & 101.007 & 425.498 & 1178.67 & 50.662 & 140.35 & 36.463 & 101.007 & 425.498 & 1178.67 & 33.776 & 93.562 \\
\hline 106 & 15.353 & 42.53 & 946.227 & 2067.11 & 28.444 & 78.792 & 76.766 & 212.649 & 298.491 & 826.845 & 14.222 & 39.396 \\
\hline 107 & 18.821 & 54.083 & 366.035 & 1051.82 & 19.371 & 55.664 & 18.821 & 54.083 & 366.035 & 1051.82 & 21.793 & 62.622 \\
\hline 108 & 27.857 & 78.692 & 565.001 & 1596.05 & 32.256 & 91.117 & 27.857 & 78.692 & 541.781 & 1530.46 & 51.608 & 145.786 \\
\hline 109 & 17.053 & 48.583 & 331.657 & 944.89 & 26.326 & 75.004 & 21.316 & 60.728 & 829.141 & 2362.23 & 31.592 & 90.006 \\
\hline 110 & 94.617 & 265.035 & 115.005 & 322.141 & 21.91 & 61.373 & 23.653 & 66.255 & 368.079 & 1031.03 & 21.654 & 60.654 \\
\hline 201 & 21.57 & 59.42 & 397.688 & 1095.56 & 25.263 & 69.594 & 20.454 & 56.347 & 318.182 & 876.535 & 55.088 & 151.756 \\
\hline 202 & 118.948 & 338.882 & 462.574 & 1317.87 & 55.088 & 156.944 & 59.474 & 169.441 & 462.574 & 1317.87 & 21.186 & 60.359 \\
\hline 203 & 147.273 & 419.58 & 400.304 & 1140.47 & 23.835 & 67.905 & 34.308 & 97.743 & 400.304 & 1140.47 & 22.486 & 64.062 \\
\hline 204 & NA & NA & NA & NA & NA & NA & 42.481 & 119.329 & 330.41 & 928.117 & 80.126 & 225.072 \\
\hline 205 & 14.421 & 42.291 & 336.453 & 986.666 & 32.049 & 93.985 & 17.299 & 50.731 & 280.397 & 822.28 & 15.669 & 45.95 \\
\hline 206 & 16.916 & 48.608 & 411.123 & 1181.39 & 17.41 & 50.028 & 21.144 & 60.759 & 328.899 & 945.111 & 35.203 & 101.157 \\
\hline 207 & 26.603 & 78.939 & 517.332 & 1535.11 & 35.203 & 104.459 & 44.339 & 131.569 & 517.332 & 1535.11 & 196.132 & 581.994 \\
\hline 208 & 21.331 & 56.882 & 331.796 & 884.788 & 19.759 & 52.69 & 28.441 & 75.843 & 331.796 & 884.788 & 21.512 & 57.364 \\
\hline 209 & 27.094 & 75.26 & 316.147 & 878.187 & 25.097 & 69.713 & 20.32 & 56.447 & 316.147 & 878.187 & 22.107 & 61.408 \\
\hline 210 & 39.777 & 118.032 & 464.202 & 1377.46 & 55.268 & 164.001 & 39.777 & 118.032 & 464.202 & 1377.46 & 18.854 & 55.947 \\
\hline 211 & 18.319 & 55.177 & 356.307 & 1073.21 & 18.854 & 56.789 & 18.319 & 55.177 & 356.307 & 1073.21 & NA & NA \\
\hline
\end{tabular}

Table 2. Predicted occlusion time, delay time and kinetic constants using calorimetric data (Hofrichter J, Ross PD, Eaton WA, 1974) [10].

\begin{tabular}{|c|c|c|c|c|c|c|c|}
\hline $\mathrm{T},{ }^{\circ} \mathrm{C}$ & Slope @f = 0.5 & $\mathrm{~T}_{1 / 2}, \min$ & $t_{D}, \min$ & $K$ & $k_{1}$ & $k_{2}$ & $k_{2} / k_{1}$ \\
\hline 15.9 & 0.006 & 925 & 307.692 & 0.024 & 0.024 & 0.103 & 4.29 \\
\hline 16.3 & 0.006 & 835 & 307.692 & 0.024 & 0.024 & 0.103 & 4.29 \\
\hline 17 & 0.007 & 675 & 181.818 & 0.028 & 0.028 & 0.1202 & 4.29 \\
\hline 17.8 & 0.006 & 512.5 & 142.857 & 0.024 & 0.024 & 0.103 & 4.29 \\
\hline 18.7 & 0.004 & 325 & 117.647 & 0.016 & 0.016 & 0.0687 & 4.29 \\
\hline 19.6 & 0.009 & 100 & 100.000 & 0.036 & 0.036 & 0.1545 & 4.29 \\
\hline 20.3 & 0.048 & 85 & 40.000 & 0.192 & 0.192 & 0.824 & 4.29 \\
\hline
\end{tabular}


Table 3. Predicted occlusion, delay time and kinetic constants birefringence data (Hofrichter J, Ross PD, Eaton WA, 1974) [10].

\begin{tabular}{cccccccc}
\hline $\mathrm{T},{ }^{\circ} \mathrm{C}$ & Slope @f $=0.5$ & $T_{1 / 2}, \min$ & $t_{D}, \min$ & $K$ & $k_{1}$ & $k_{2}$ & $k_{2} / k_{1}$ \\
\hline 18 & 0.002 & 250 & 142.857 & 0.008 & 0.008 & 0.0343 & 4.29 \\
20 & 0.016 & 96.25 & 62.500 & 0.064 & 0.064 & 0.2747 & 4.29 \\
22.5 & 0.019 & 16.25 & 11.976 & 0.076 & 0.076 & 0.3262 & 4.29 \\
25 & 0.173 & 5.75 & 7.692 & 0.692 & 0.692 & 2.97 & 4.29 \\
30 & 3.467 & 0.85 & 0.930 & 13.868 & 13.868 & 59.5193 & 4.29 \\
\hline
\end{tabular}

\subsection{Discussion}

The most striking result obtained is the constant $k$ ratios for both in vivo and in vitro model validation. The $k$ ratios show by how much the secondary nucleation is faster than the primary nucleation. For in vitro polymerization, the $k$ ratios gave a constant value of 4.29 irrespective of the monitoring technique used. However, having constant $k$ ratios from in vitro experiments suggest that the mechanisms of nucleation deduced sheds light on the in vivo polymerization of HbS in SCD patients. The $\mathrm{k}$ ratios for each SCD patient in all the arteries investigated was observed to be constant also. The severity of the symptoms and presentation of sickle cell disease can therefore be predicted with the $k$ ratios. The $k$ ratios, therefore, are indicative of patients' susceptibility to more frequent occlusive events/periodic pain cycles but seems to be heightened by higher hematocrits as postulated by Stuart (Stuart MJ, Nagel RL., Oct, 2004) [11].

No single parameter from the blood indices can be traced to the results obtained but a combination of the data from both the blood indices and Transcranial Doppler appears useful. Although blood flow velocities observed could not be immediately linked directly with any of the parameters measured yet small SD (red cell sizes) coupled with short vessel lengths gave very low velocities and the Transcranial Doppler (TCD) results showed more turbulence in the flow of SCD patients as compared to the control participants. Velocities of blood flow in the arteries were at least 1.5 times the values recorded in the control participants, which is at least $50 \%$ higher than for the control participants.

Since the delay time appears to be critical in the pathophysiology of sickle cell disease, SCD, it can be prolonged by either increasing the ability of the red cells to oxygenate or increasing the crowding agents, like $\mathrm{Hb} \mathrm{F}$, in the red cell.

\section{Conclusions}

The major finding in this study is the constant $k_{2} / k_{1}$ ratios peculiar to individuals participating in the in vivo data collection and the in vitro literature data. This ratio suggests an independence on the environment and reaction path and also, gives insight into the degree of expression of the symptoms of SCD with higher values reflecting greater propensity to pain crisis. Comparing the in vivo and in vitro results, higher rate constant ratios were obtained for data collected for in vivo patients. This may be largely due to the temperature differences in the two cases.

Therefore, it is recommended that the constancy of the model generated $k_{2} / k_{1}$ ratios with the diameter of the blood vessel be used in the pain management of SCD patients.

\section{References}

[1] Pauling, L., Itano, H.A., Singer, S.J. and Wells, I.C. (1949) Sickle Cell Anemia: A Molecular Disease. Science, 10, 543-548. http://dx.doi.org/10.1126/science.110.2865.543

[2] Petsko, G.A. and Ringe, D. (2004) Protein Structure and Function. New Science Press Ltd., UK.

[3] FAO (1990) Conducting Small-Scale Nutrition Surveys: A Field Manual. Rome.

[4] Magnanni, R. (1997) Sampling Guide. IMPACT Food Security and Nutrition Monitoring Project. Arlington, Va.

[5] UNICEF (1995) Monitoring Progress towards the Goals of the World Food Summit for Children: A Practical Hand- 
book for Multiple Indicator Surveys. New York.

[6] Boudart, M. (1968) Kinetics of Chemical Processes. Prentice-Hall, INC.

[7] Susu, A.A. (1997) Chemical Kinetics and Heterogeneous Catalysis. CJC Publishers Nig. Ltd.

[8] Susu, A.A. and Kunugi, T. (1980) A Novel Pyrolytic Decomposition of n-Erosane with Synthesis gas and $\mathrm{K}_{2} \mathrm{CO}_{3} \mathrm{Cat}_{-}$ alyzed Shift Reaction. I \& EC Proc. Des. Dev., 19, 693-699. http://dx.doi.org/10.1021/i260076a031

[9] Charm, S.E. and Kurland, G.S. (1974) Blood Flow and Microcirculation. John Wiley \& Sons.

[10] Hofrichter, J., Ross, P.D. and Eaton, W.A. (1974) Kinetics and Mechanism of Deoxyhemoglobin S Gelation: A New Approach to Understanding Sickle Cell Disease. Proc. Natl. Acad. Sci. USA, 71, 4864-4868. http://dx.doi.org/10.1073/pnas.71.12.4864

[11] Stuart, M.J. and Nagel, R.L. (2004) Sickle-Cell Disease. 364. www.thelancet.com 